\title{
Comparative analysis of adaptive immune responses following experimental infections of cattle with bovine viral diarrhoea virus- 1 and an Asiatic atypical ruminant pestivirus
}

\author{
Victor Riitho ${ }^{\mathrm{a}, \mathrm{b}, \mathrm{f}}$, Magdalena Larska ${ }^{\mathrm{c}, \mathrm{d}}$, Rebecca Strong ${ }^{\mathrm{a}}$, S. Anna La Rocca ${ }^{\mathrm{a}}$, Nicolas Locker ${ }^{\mathrm{b}}$, \\ Stefan Alenius ${ }^{\mathrm{c}}$, Falko Steinbach ${ }^{\mathrm{a}, \mathrm{b}}$, Lihong Liu ${ }^{\mathrm{c}}$, Åse Uttenthal ${ }^{\mathrm{e}}$, Simon P. Graham ${ }^{\mathrm{a}, \mathrm{b}, *}$ \\ ${ }^{a}$ Animal and Plant Health Agency, Woodham Lane, Addlestone KT15 3NB, UK \\ ${ }^{\mathrm{b}}$ Faculty of Health and Medical Sciences, University of Surrey, Guildford GU2 7XH, UK \\ ${ }^{\mathrm{c}}$ Swedish University of Agricultural Sciences, Box 7054, SE-750 07 Uppsala, Sweden \\ ${ }^{\mathrm{d}}$ National Veterinary Research Institute, Al. Partyzantów 57, 24-100, Puławy, Poland \\ e National Veterinary Institute, Technical University of Denmark, Lindholm, DK-4771 Kalvehave, Denmark \\ ${ }^{\mathrm{f}}$ International Livestock Research Institute, P.O. Box 30709, Nairobi 00100, Kenya ${ }^{1}$
}

\section{A R T I C L E I N F O}

\section{Article history:}

Received 11 October 2017

Received in revised form 28 May 2018

Accepted 6 June 2018

Available online 12 June 2018

\section{Keywords:}

BVDV

Atypical pestivirus

Immune responses

Cross-reactivity

\begin{abstract}
A B S T R A C T
Atypical ruminant pestiviruses are closely related to the two bovine viral diarrhoea virus (BVDV) species, BVDV-1 and BVDV-2. While there is evidence of cross-protective immune responses between BVDV-1 and BVDV-2, despite antigenic differences, there is little information on the antigenic cross-reactivity with atypical ruminant pestiviruses. The aim of this study was therefore to assess the specificity of antibody and $\mathrm{T}$ cell responses induced by experimental infection of calves with BVDV-1 strain Ho916 Th/04_KhonKaen (TKK), an Asiatic atypical ruminant pestivirus, or co-infection with both viruses. Homologous virus neutralization was observed in sera from both single virus infected and co-infected groups, while cross-neutralization was only observed in the TKK infected group. T cell IFN- $\gamma$ responses to both viruses were observed in the TKK infected animals, whereas Ho916 infected calves responded better to homologous virus. Specifically, IFN- $\gamma$ responses to viral non-structural protein, NS3, were observed in all infected groups while responses to viral glycoprotein, E2, were virus-specific. Broader antigen-specific cytokine responses were observed with similar trends between inoculation groups and virus species. The limited $\mathrm{T}$ cell and antibody immune reactivity of Ho916 inoculated animals to TKK suggests that animals vaccinated with current BVDV-1-based vaccines may not be protected against atypical ruminant pestiviruses.
\end{abstract}

Crown Copyright @ 2018 Published by Elsevier Ltd. All rights reserved.

\section{Introduction}

The Pestivirus genus within the Flaviviridae family of single stranded positive-sense RNA viruses comprises four recognized species: BVDV-1, BVDV-2, classical swine fever virus (CSFV) and border disease virus (BDV) [1]. Additionally, eight groups of unclassified pestiviruses have been discovered: giraffe, pronghorn antelope, Bungowannah, atypical ruminant, sheep, bat, rat and atypical porcine pesitiviruses [2,3]. Atypical ruminant pestiviruses, informally referred to as BVDV-3 or HoBi-like viruses, which form a

\footnotetext{
* Corresponding author at: The Pirbright Institute, Ash Road, Woking GU24 0NF, UK.

E-mail address: simon.graham@pirbright.ac.uk (S.P. Graham).

1 Present address.
}

monophyletic clade with a sister relationship to BVDV-1 and -2 , have been detected in contaminated foetal bovine serum (FBS) and in natural infections in cattle and buffalo [4,5]. While the evidence suggests atypical bovine pestiviruses are present in cattle in South America, Southeast Asia and Europe, there is a need for broader surveillance to assess the extent of their geographic distribution and the possible impact on current and future BVDV control programs.

Genetic and antigenic diversity of bovine pestiviruses may pose a significant challenge to BVDV diagnosis and vaccination. Diagnosis by detection of viral material or through quantification of virus-specific immune responses may be hampered by the variability within these targets making it difficult to recognise divergent or atypical viruses [6]. Furthermore, limited virus cross-neutralization and failure in detection of seroconversion to 
atypical pestiviruses using commercial ELISA (based on BVDV-1) is evidence of significant antigenic diversity between atypical pestiviruses and BVDV-1 and -2 [7]. This also raises questions regarding the actual distribution of atypical pestiviruses and potential efficacy of existing BVDV vaccines against these divergent viruses. Whilst clinical cross-protection against BVDV-2 challenge has been reported using BVDV-1 vaccines, vaccination failure has been attributed to virus diversity and inadequate protection from foetal infection, postnatal infection and virus shedding $[8,9]$. In future, vaccines may have to be designed with protection against BVDV-1 and - 2 species and sub-genotypes as well as atypical bovine pestiviruses in mind [9]. This may be achieved by inclusion of different virus genotypes/species, identification of targets of cross-protective immune responses, or by the use of speciesspecific vaccines.

This study, conducted as part of a study comparing the infection kinetics and pathogenesis of calves experimentally infected with a BVDV-1a strain (Ho916), an Asiatic atypical ruminant pestivirus (Th/04_KhonKaen) or co-infection with both viruses, aimed to assess whether antibody and $\mathrm{T}$ cell responses induced by one virus were capable of responding to the heterologous virus and to determine if the immunodominant antigens, E2 glycoprotein and nonstructural protein NS3, were targets of cross-reactive or distinct species-specific responses.

\section{Materials and methods}

\subsection{Viruses and animals}

The study used the non-cytopathic BVDV-1a strain Ho916, isolated from a clinically severe outbreak of BVD in the United Kingdom [10,11], and the non-cytopathic atypical bovine pestivirus Th/04_KhonKaen (TKK), which was recovered from a dairy calf in Thailand [12,13]. Both viruses were propagated in low passage foetal bovine turbinate ( $\mathrm{fBT}$ ) cells and virus titres determined as the median $(50 \%)$ tissue culture infectious dose $\left(\mathrm{TCID}_{50}\right)$ using the Spearman-Kärber method following immunoperoxidase staining [14]. Twenty 3-5 month old, BVDV-naïve Holstein calves were randomly assigned into 4 groups of 5 animals. The calves were confirmed to be BVDV antibody and antigen negative to rule out pre-exposure and persistent infection. Calves were inoculated with $10^{5}$ TCID $_{50}$ of Ho916, TKK or a mixture of both viruses (Ho916/ TKK). Each animal was inoculated with the appropriate virus or viruses diluted in $10 \mathrm{~mL}$ EMEM (Invitrogen, Thermo Fisher Scientific, Paisley, UK) by intranasal $(5 \mathrm{~mL})$ and intramuscular $(5 \mathrm{~mL})$ routes simultaneously in $1 \mathrm{~mL}$ of EMEM (Invitrogen, Thermo Fisher Scientific, Paisley, UK). Control animals (Controls) were similarly inoculated with $10 \mathrm{~mL}$ of EMEM only. Assessment of clinical scores including rectal temperature and sampling of blood and nasal secretions was conducted up to 45 days post-infection (dpi). The study was approved by the Ethics Committee for Animal Experiments by the Ministry of Science and Higher Education in Poland (No. 79/2010) and details of the procedures and outcome of the experimental infection are previously described [15]. In brief, milder clinical signs were observed in the TKK group and coinfection resulted in prolonged pyrexia in comparison to the single infection groups. Similar courses of viraemia were measured in all three infected groups. Simultaneous detection of both viruses in blood and nasal swabs was observed in the co-infected group. All inoculated groups seroconverted two weeks after inoculation as assessed by serum neutralisation of homologous virus. In addition to Ho916 and TKK viruses, BVDV-1a C24V (C24V) [16], BVDV-2 502643-02-UK (BVDV-2) [17] and border disease virus $1 \mathrm{~S} 137 / 4$ (BDV-1) [18] reference strains were used to assess serological cross reactivity.

\subsection{Detection of antibody responses}

Competitive ELISAs were used for the detection of antibodies against BVDV-1 NS3 (SVANOVIR ${ }^{\circledR}$ BVDV p80, Svanova Biotech AB, Uppsala, Sweden) and E2 (VDPro ${ }^{\circledR}$ BVDV, Jeno Biotech, Chuncheon, Republic of Korea) according to the manufacturer's instructions. Virus neutralizing antibody titres (VNT) in pooled serum samples for each inoculation group at $42 \mathrm{dpi}$ were determined against Ho916, TKK, C24V, BVDV-2 and BDV-1 as described previously [19]. VNT were calculated as reciprocal of the highest serum dilution that inhibited virus growth by $50 \%$ using the SpearmanKärber method and expressed on a $\log _{2}$ scale [14]. The extent of cross-reactivity was calculated from log transformed VNTs as a percentage of heterologous virus neutralisation compared to homologous neutralisation [20].

\subsection{Synthetic pestiviral peptides}

Predicted amino acid sequences of Ho916 E2 and NS3 (GenBank MH379638) were aligned against the reference strain C24V (GenBank AF091605.1) and Th/04_KhonKaen (GenBank FJ040215.1) using the ClustalW algorithm on MegAlign (DNAStar Lasergene 9 Core Suite, Madison, WI, USA). Based on the divergence of the E2 and conservation of NS3 between the three viruses, overlapping pentadecamer peptides offset by four residues (JPT Peptide Technologies, Berlin, Germany) were synthesised covering the complete E2 protein from C24V, Ho916 and TKK (Fig. 1), and the NS3 protein of C24V only (Fig. 2). Lyophilised peptides were dissolved in $0.1 \mathrm{M}$ HEPES pH 7.4-buffered $40 \%$ acetonitrile and pooled to represent the respective E2 and NS3 proteins. Peptide pools were diluted in RPMI-1640 medium supplemented with $2 \mathrm{mM}$ L-glutamine, 20 $\mathrm{mM}$ HEPES, $100 \mathrm{U} / \mathrm{mL}$ penicillin, $100 \mu \mathrm{g} / \mathrm{mL}$ streptomycin, $50 \mathrm{mM}$ 2-mercaptoethanol (all Invitrogen) and 10\% FBS (Autogen Bioclear, Calne, UK) (cRPMI).

\subsection{Ex vivo stimulation of peripheral blood mononuclear cells and assessment of cytokine responses}

Peripheral blood mononuclear cells (PBMC) from calves were isolated weekly from heparinised blood by density centrifugation using Histopaque-1077 (Sigma, Poole, UK), suspended in cRPMI, and added $5 \times 10^{5}$ cells/well to 96 well round-bottom plates. Cells were stimulated in duplicate wells with $100 \mu$ l of peptide pools representing the E2 and NS3 proteins at $1 \mu \mathrm{g} / \mathrm{mL}$ or viruses at a multiplicity of infection of 1 . Medium or mock-infected fBT supernatant and pokeweed mitogen (Sigma) were used as negative and positive controls respectively. The cells were incubated at $37{ }^{\circ} \mathrm{C}$ for $48 \mathrm{~h}$ after which cell-free supernatants were harvested after centrifugation and stored at $-80^{\circ} \mathrm{C}$ until analysed. The release of IFN- $\gamma$ in culture supernatants was measured using a bovine IFN$\gamma$ ELISA as previously described [21]. IFN- $\gamma$, IL-4, IL-10 and MIP$1 \beta$ were simultaneously quantified using a custom-designed multi-array electrochemiluminescence detection assay (Meso Scale Discovery, Gaithersburg, USA) as described [22].

\subsection{Data analysis and statistics}

Graphical and statistical analysis was performed using GraphPad Prism 7.01 (GraphPad Software, La Jolla, USA). Data was represented as means with standard error of means (SEM) shown to indicate the uncertainty around the estimate of the group means. Two-way ANOVA followed by a Dunnett's or Tukey's multiple comparison tests were used for the analysis of virus strain specific effects on antibody and cytokine responses in the different infection groups. $P$ values are indicated on figures. 


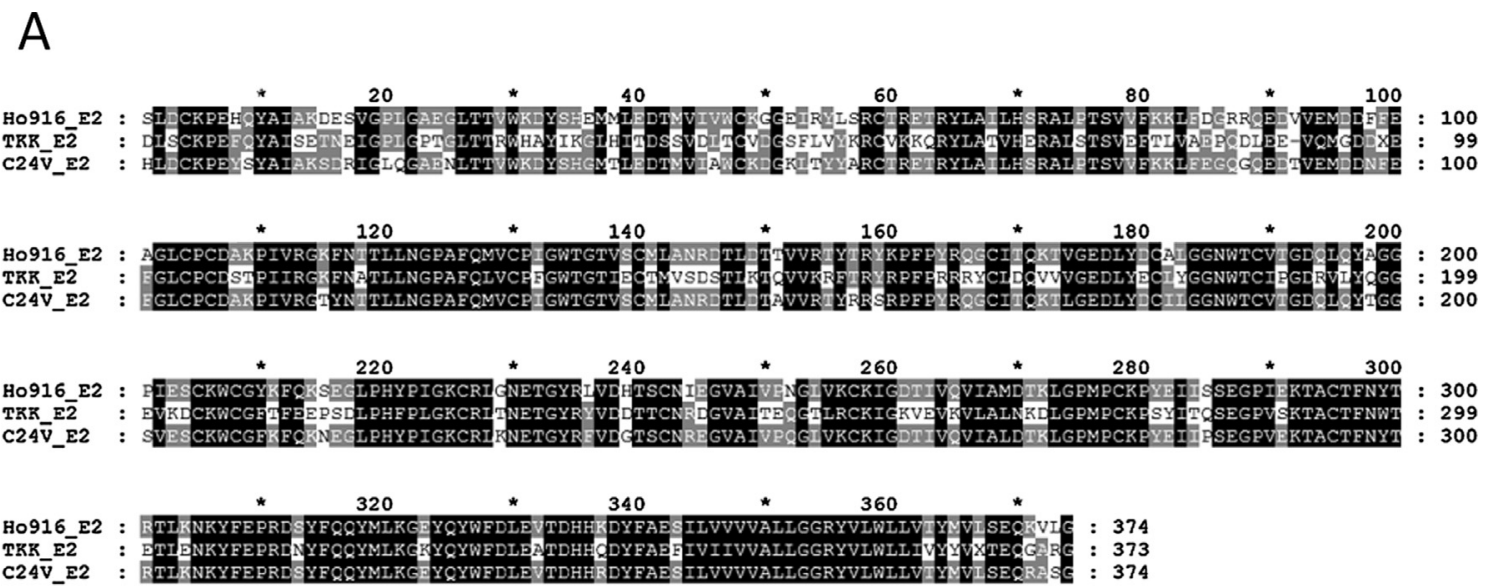

B

\begin{tabular}{|c|c|c|c|c|c|}
\hline & \multicolumn{5}{|c|}{ \% Identity } \\
\hline \multirow{5}{*}{ 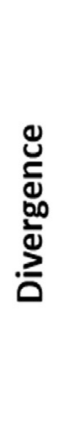 } & & Ho916 & TKK & C24V & \\
\hline & Ho916 & & 58.6 & 86.6 & Ho916 \\
\hline & TKK & 58.6 & & 60.2 & TKK \\
\hline & $\mathrm{C} 24 \mathrm{~V}$ & 14.8 & 15.3 & & C24V \\
\hline & & Ho916 & TKK & $\mathrm{C} 24 \mathrm{~V}$ & \\
\hline
\end{tabular}

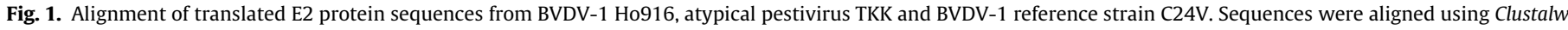

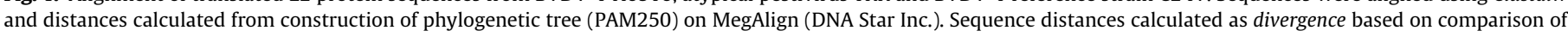

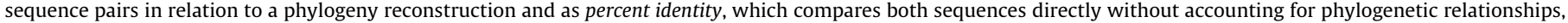
are presented in the tables.

\section{Results}

\subsection{Assessment of virus-specific antibody responses}

The Ho916 and Ho916/TKK infected groups mounted E2 specific antibody response at $14 \mathrm{dpi}$ (Fig. 3A) as determined by a competitive BVDV ELISA. While the E2 specific antibody levels in the TKK group increased from $14 \mathrm{dpi}$ they were not above the cut-off until 21 dpi. The E2 specific antibody levels of the Ho916 and Ho916/ TKK groups were significantly higher than in the TKK group from 14 dpi. The Ho916 and Ho916/TKK groups showed NS3 antibody responses (Fig. 3B) from $21 \mathrm{dpi}$ compared to the control group, whilst the TKK group responses were significantly higher compared to the controls from 28 dpi but did not exceed the diagnostic cut-off for assay positivity until $35 \mathrm{dpi}$. Ho916 group responses were significantly greater than the TKK group at 21 and $28 \mathrm{dpi}$, whereas, Ho916/TKK antibody levels were greater than TKK between 14 and $35 \mathrm{dpi}$. There were no significant differences in NS3 responses between Ho916 and Ho916/TKK groups. Homologous neutralization of both Ho916 and TKK viruses was observed from $14 \mathrm{dpi}$ in the single and co-infected groups. However, neutralization of Ho916 virus by sera from TKK inoculated cattle only occurred at low titres at $42 \mathrm{dpi}$ while low titres of TKK- neutralizing antibodies was observed in Ho916 inoculated animals from $21 \mathrm{dpi}$. We further assessed the cross-neutralising properties of these sera by comparing neutralisation of the inoculated viruses, and reference strains
BVDV-1a C24V, BVDV-2 50263-02-UK and BDV-1 S137/4 (Fig. 4A). Ho916 virus neutralization titres in both Ho916 and Ho916/TKK group sera were higher than the TKK group sera and a similar pattern was observed against C24V and BDV-1 but with lower titres. In contrast, TKK virus was neutralized by TKK and Ho916/TKK inoculated group sera but not by the Ho916 inoculated animals' sera. Neutralization of BVDV-2 was observed at low titres in all inoculated groups with the lowest titres in sera from Ho916 inoculated animals. Ho916 and Ho916/TKK group sera better neutralized BDV compared to sera from the TKK inoculated animals. Assessment of cross-neutralisation, calculated as the percentage of heterologous versus homologous neutralization, showed that despite exhibiting weaker homologous neutralization, TKK group sera showed comparatively good neutralization of Ho916, C24V and BVDV-2 (Fig. 4B). Ho916 group sera showed crossneutralization of $\mathrm{C} 24 \mathrm{~V}$ albeit with lower titres compared to homologous neutralization. While cross-neutralization of all other viruses by sera from the Ho916 inoculated animals was considerably less than homologous virus neutralization. Cross-neutralization of BDV-1 by sera from all inoculation groups was poor.

\subsection{Assessment of virus-specific $T$ cell responses}

In comparison to uninfected controls, significant ex vivo PBMC IFN- $\gamma$ responses were observed against Ho916 and TKK viruses from 35 dpi (Fig. 5). PBMC from the Ho916 inoculated group 
A

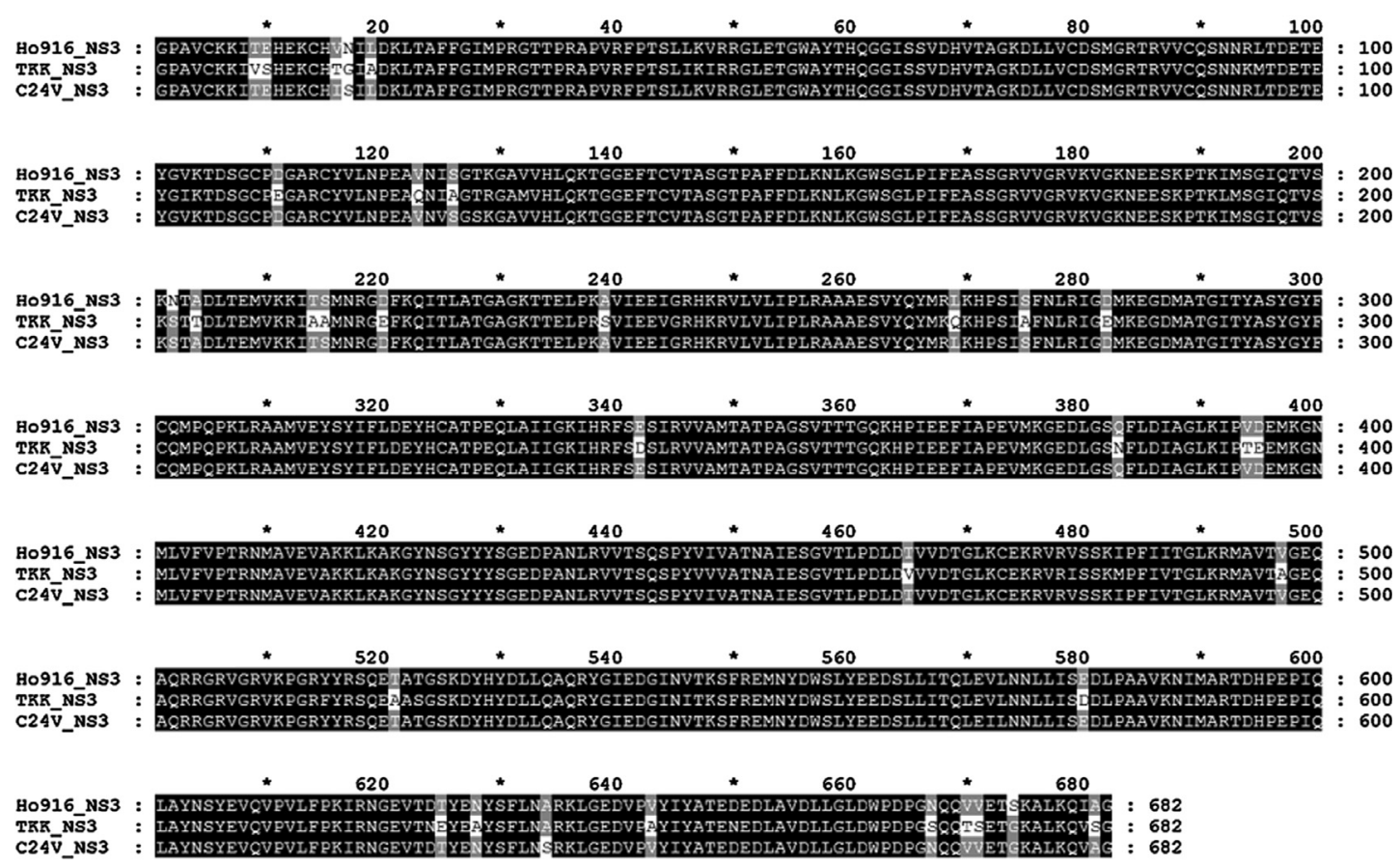

B

\begin{tabular}{|c|c|c|c|c|c|}
\hline & \multicolumn{5}{|c|}{$\%$ Identity } \\
\hline \multirow{5}{*}{ 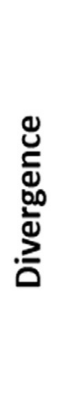 } & & Ho916 & TKK & C24V & \\
\hline & Ho916 & & 91.8 & 98.5 & Ho916 \\
\hline & TKK & 8.7 & & 91.8 & TKK \\
\hline & $\mathrm{C} 24 \mathrm{~V}$ & 1.5 & 8.7 & & $\mathrm{C} 24 \mathrm{~V}$ \\
\hline & & Ho916 & TKK & C24V & \\
\hline
\end{tabular}

Fig. 2. Alignment of translated NS3 protein sequences from BVDV-1 Ho916, atypical pestivirus TKK and BVDV-1 reference strain C24V. Sequences were aligned using Clustalw and distances calculated from construction of phylogenetic tree (PAM250) on MegAlign (DNA Star Inc.). Sequence distances calculated as divergence based on comparison of sequence pairs in relation to a phylogeny reconstruction and as percent identity, which compares both sequences directly without accounting for phylogenetic relationships, are presented in the tables.

stimulated with Ho916 virus showed statistically significant IFN- $\gamma$ responses on $42 \mathrm{dpi}$, whilst responses in the TKK group significant on 35 and 42 dpi. Responses to Ho916 virus in the Ho916/TKK group were less than in the other two groups and were not statistically significant. Responses to the TKK virus on the other hand, were significant in both the TKK and Ho916/TKK groups at 35 and 42 dpi. Responses to the TKK virus by PBMC from the Ho916 group were not statistically significant. IFN- $\gamma$ responses to E2 peptide pools at 42 dpi followed similar trends to the responses to virus (Fig. 6A). IFN- $\gamma$ responses to the E2 peptide pool representing Ho916 virus (E2-Ho916) were observed in the Ho916 and Ho916/ TKK groups but not in the TKK group. Responses to E2-TKK peptide pool were seen in the TKK and Ho916/TKK groups, albeit only statistically significant in the TKK group. IFN $-\gamma$ responses to the NS3 peptide pool were observed in all infected groups and were statistically significant in the Ho916 and TKK infected groups. Broader cytokine responses including IL-10, IL- 4 and MIP- $1 \beta$ were observed following virus and peptide stimulation (Fig. 6B-D). TKK infected animals were observed to elicit a broad cytokine response following stimulation with homologous virus, as well as heterologous virus. Significant quantities of IL-4, IL-10, and MIP- $1 \beta$ were secreted by PBMC from TKK infected animals following TKK and Ho916 stimulation. With the exception of MIP-1 $\beta$, the other cytokines were not secreted to significant levels in the Ho916 infected animals following stimulation with the TKK virus. While IL-4 production was observed at lower levels in the co-infected animals in response to both viruses, IL-10, and MIP- $1 \beta$ were secreted in significant levels in this group following TKK stimulation. Significant IL4 responses to the E2-Ho916 were observed in the Ho916 virus and co-infected groups and to the NS3 C24V peptide pool in the coinfected group but not against E2-TKK in any of the infected groups. IL-10 responses against NS3 and E2-TKK peptide pools 
A

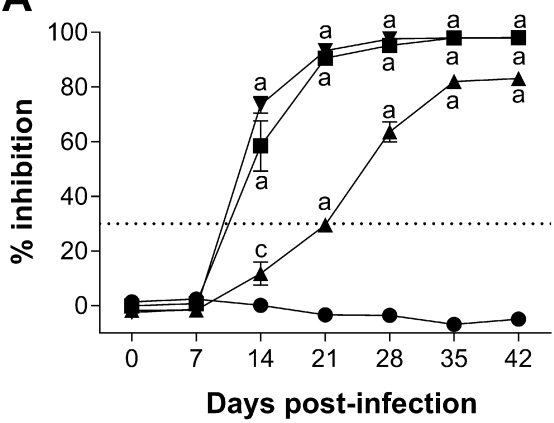

B

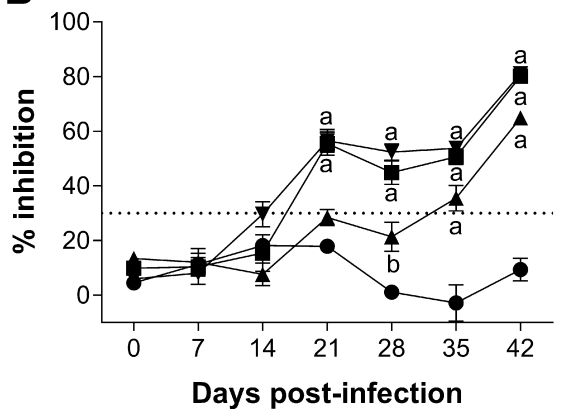

- Control

- Ho916

$\triangle$ TKK

V Ho916/TKK

Fig. 3. Antigen-specific antibody responses of calves following experimental infection with BVDV-1 Ho916, atypical bovine pestivirus TKK, co-infection with both (Ho916/TKK) or uninfected controls. Longitudinal antibody responses were measured by competitive ELISA against BVDV-1 (A) E2 and (B) NS3 proteins. Antibody levels were calculated as \% inhibition of antigen specific $m A b$ binding, and dashed lines denote positivity cut-off for the respective assays. Data represented as means \pm SEM; a two-way ANOVA with Dunnett's post-test was performed and statistical significances between infected and control groups are described by ' $a$ ' $\mathrm{p}<0.0001$, 'b' $\mathrm{p}<0.001$, and 'c' $\mathrm{p}<0.01$.

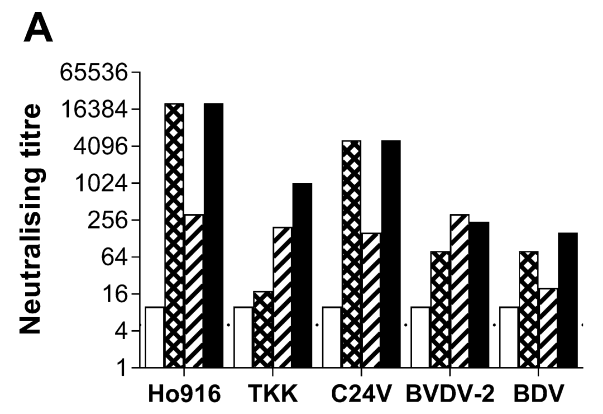

B

\begin{tabular}{|c|}
\hline $\begin{array}{l}\square \text { Control } \\
\text { Ho916 } \\
\mathbb{\text { TKK }} \\
\text { Ho916/TKK }\end{array}$ \\
\hline
\end{tabular}

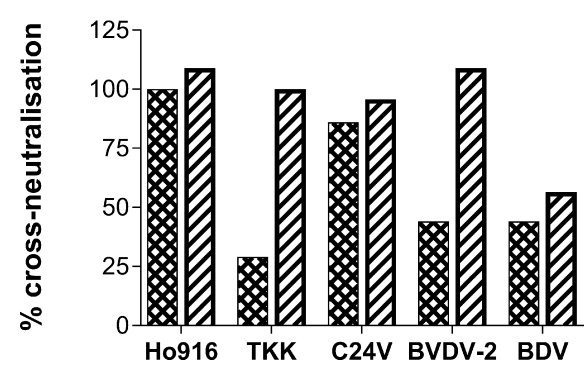

Fig. 4. Homologous and heterologous virus neutralising antibody titres of calves following experimental infection with BVDV-1 Ho916, atypical bovine pestivirus TKK, co-infection with both (Ho916/TKK) or uninfected controls. (A) Virus neutralising antibody titres of serum pools collected at $42 \mathrm{dpi}$ from the different infection groups against Ho916, TKK, BVDV-1a Oregon C24V (C24V), BVDV-2 502643-02-UK (BVDV-2) and BDV-1 S137/4 (BDV). (B) Cross-neutralization of heterologous virus was calculated as a percentage of homologous neutralization by Ho916 or TKK inoculated cattle.

A

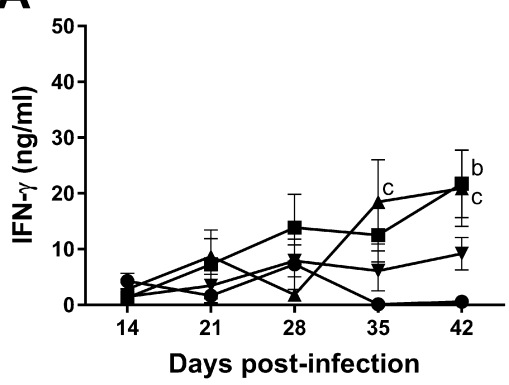

B

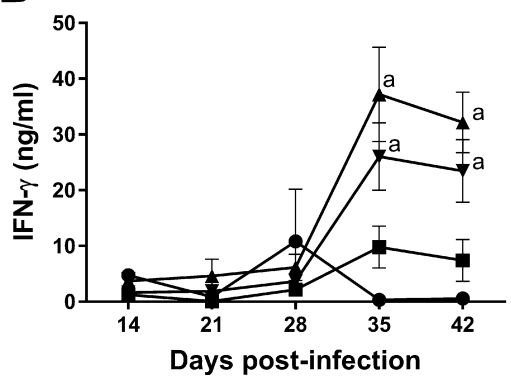

Fig. 5. Longitudinal virus-specific IFN- $\gamma$ responses of calves following experimental infection with BVDV-1 Ho916, atypical bovine pestivirus TKK, co-infection with both (Ho916/TKK) or uninfected controls. Longitudinal PBMC IFN- $\gamma$ responses following stimulation with (A) Ho916 and (B) TKK viruses were measured by ELISA and adjusted for mock stimulated background responses. Data represented as group means $\pm \mathrm{SEM}$; a two-way ANOVA with Dunnett's post-test was performed and statistical significances between infected and control groups are described by ' $a$ ' $\mathrm{p}<0.0001$, 'b' $\mathrm{p}<0.001$, and 'c' $\mathrm{p}<0.01$.

were observed in the Ho916 infected group, and against E2-TKK peptide pool in the TKK infected group while the co-infected group had significant responses to both E2 peptide pools and NS3. MIP-1 $\beta$ responses were significant in all infected groups to E2-Ho916 and NS3 and to E2-TKK in the TKK and co-infected groups.

\section{Discussion}

Recent disease outbreaks within the EU, in Asia and South America caused by atypical ruminant pestiviruses have highlighted a group of viruses that have thus far received little attention. Comparative analyses of antigenic relationships between atypical ruminant pestiviruses and BVDV-1 and BVDV-2 point towards the need for improved diagnostics and reformulation of current vaccines for the detection and control of these new viruses [7]. This study aimed to increase the understanding of antigenic diversity on both $\mathrm{T}$ cell and humoral responses to BVDV-1 and atypical pestiviruses.

In this study, both BVDV-1 E2 and NS3 specific antibody responses were detected in all infected groups 14-21 dpi postinfection albeit to a lesser extent in the TKK group. This raises questions about the sensitive detection of antibodies against atypical ruminant pestiviruses, such as TKK, by diagnostic ELISAs designed to detect antibodies against BVDV-1 [6]. This is supported by the limited detection of antibodies to a European atypical bovine pestivirus, Italy-1/10-1, using a commercial BVDV-1based ELISA, despite high neutralization titres [23]. In the present study, NS3 antibodies were better detected than antibodies against the less conserved E2 protein. It has recently been proposed that diagnostics for BVDV and atypical pestiviruses should be based on $\mathrm{NS} 2 / 3$ or $\mathrm{E}^{\mathrm{rns}}$ as they potentially possess more cross-reactive epitopes, while assays targeting E2 may allow discrimination of pestivirus species [7]. Homologous virus neutralization was observed in all infected groups, yet cross-neutralization was only 


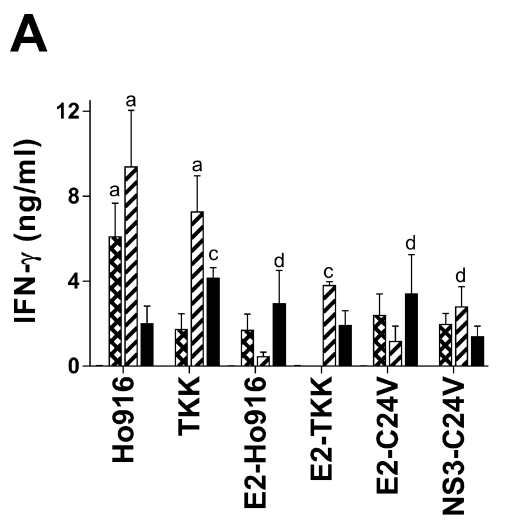

B
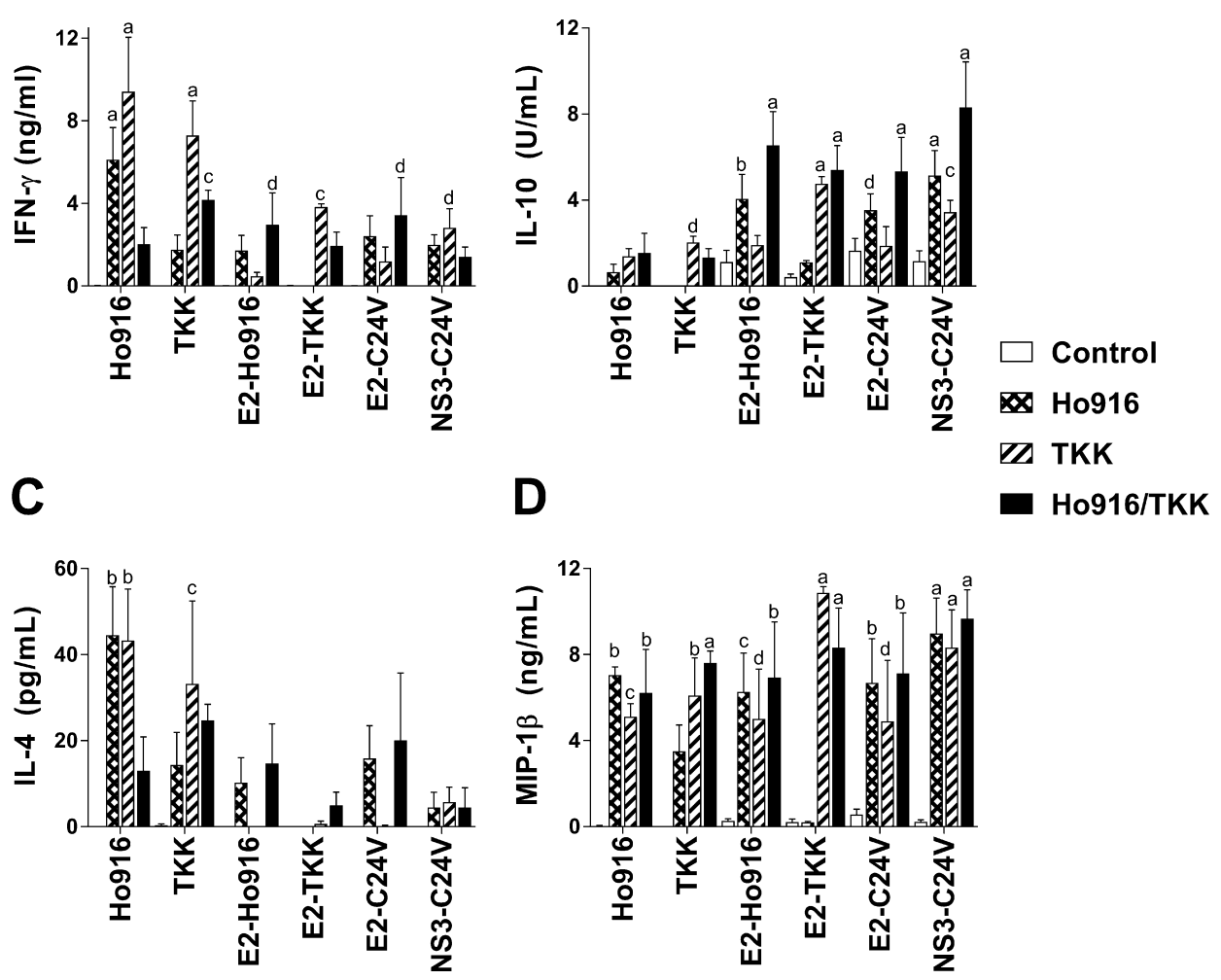

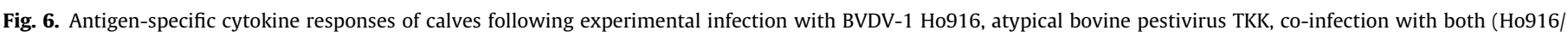

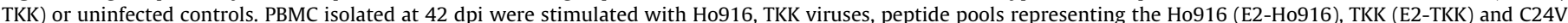

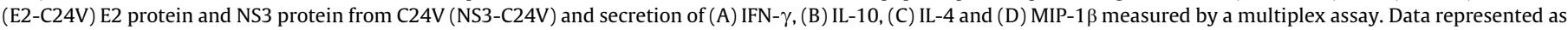

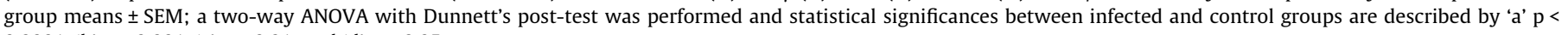
0.0001 , 'b' $p<0.001$, 'c' $p<0.01$, and 'd' $p<0.05$.

observed against the Ho916 virus in the TKK infected group, whose sera was able to neutralize the Ho916 virus almost to the same extent as the homologous virus. The relatively low homologous neutralizing antibody titres against TKK however, are suggestive of an inferior antibody response compared to the more robust titres in the Ho916 infected and co-infected groups. While similar patterns of cross-neutralisation were observed between BVDV-1 and atypical pestivirus sera against BDV-1, atypical pestivirus serum appeared to neutralize BVDV-2 better than BVDV-1 serum. Limited cross neutralization activity, alluding to significant antigenic differences between BVDV-1 and -2 and atypical ruminant pestiviruses have been previously reported [13,24,25].

A degree of cross-reactivity of $\mathrm{T}$ cell responses in TKK infected animals after re-stimulation with Ho916 virus was observed. However, IFN- $\gamma$ responses to TKK virus were low in the Ho916 infected group. IFN- $\gamma$ responses to E2 peptide pools were virus species specific whilst responses to the more conserved NS3 target appear to be maintained, to different extents, in all the infected groups. Broader cytokine responses to E2 and NS3 peptide pools followed similar trends to IFN- $\gamma$. Responses to E2-Ho916 were not observed in the TKK infected group, with the exception of MIP-1 $\beta$. Similarly, responses to E2-TKK were, by large, absent in the Ho916 group, while as observed with IFN- $\gamma$, a number of cytokine responses were observed to the NS3 peptide pool in all the infected groups. These observations, taken together, point towards E2 responses being virus specific, with responses to the NS3 antigen being better conserved between the viruses.

The limited $\mathrm{T}$ cell and antibody immune reactivity of Ho916 inoculated animals to Th/04_KhonKaen suggests that animals vaccinated with BVDV-1-based vaccines may not be protected against atypical ruminant pestiviruses. There is discussion on whether atypical ruminant pestiviruses should be recognized as a third BVDV species and if so, what implications this might have, from a regulatory point of view, for currently licensed BVD diagnostics and vaccines as well as for the BVDV control programmes and eradication status of countries and regions [26]. There is increasing evidence of the clinical and epidemiological relevance of these emerging viruses with clinical disease following experimental infection in cattle and sheep and reports of natural infections in Southeast Asia, South America and Europe [5]. Antigenic variation between BVDV-1 and -2 species has already led to the inclusion of BVDV-2 strains in some vaccines [27], and this needs to be considered in the case of atypical ruminant pestiviruses. Vaccine efficacy studies should also include challenge with heterotypic BVDV isolates as well as novel antigenically distinct variants [28].

\section{Acknowledgments}

This work was supported by European Union FP6 Network of Excellence EPIZONE (Contract no. FOOD-CT-2006-016236) and project OD0345 from the Department for the Environment, Food and Rural Affairs (DEFRA), UK. The authors would like to thank Prof. Mirosław P. Polak and the technical staff at the National Veterinary Research Institute, Puławy, Poland, where the animal experiment was conducted, the Mammalian Virus Investigation Unit staff at the Animal and Plant Health Agency, Addlestone, UK, and Prof. Sandor Belak, Swedish University of Agricultural Sciences (SLU)/National Veterinary Institute (SVA), Uppsala, Sweden. 


\section{Conflicts of interest}

None.

\section{References}

[1] Simmonds P, Becher P, Collett MS, Gould EA, Heinz FX, Meyers G, et al. Family flaviviridae. In: King AMQ, Adams MJ, Carstens EB, Lefkowitz EJ, editors. Virus taxonomy: ninth report of the international committee on taxonomy of viruses. San Diego: Academic Press; 2011. p. 1003-20.

[2] ICTV ICoToV. Genus: pestivirus - flaviviridae - positive sense rna viruses international committee on taxonomy of viruses (ICTV); 2017. <https://talk. ictvonline.org/ictv-reports/ictv_online_report/positive-sense-rna-viruses/w/ flaviviridae/361/genus-pestivirus>.

[3] Smith DB, Meyers G, Bukh J, Gould EA, Monath T, Scott Muerhoff A, et al. Proposed revision to the taxonomy of the genus Pestivirus, family Flaviviridae. J Gen Virol 2017;98:2106-12.

[4] Bauermann FV, Ridpath JF. HoBi-like viruses-the typical 'atypical bovine pestivirus'. Anim Health Res Rev 2015;16:64-9.

[5] Decaro N, Lucente MS, Losurdo M, Larocca V, Elia G, Occhiogrosso L, et al. HoBilike pestivirus and its impact on cattle productivity. Transboundary Emerg Dis 2016;63:469-73.

[6] Larska M, Polak MP, Liu L, Alenius S, Uttenthal A. Comparison of the performance of five different immunoassays to detect specific antibodies against emerging atypical bovine pestivirus. J Virol Methods 2013:187:103-9.

[7] Bauermann FV, Flores EF, Ridpath JF. Antigenic relationships between Bovine viral diarrhea virus 1 and 2 and HoBi virus: possible impacts on diagnosis and control. J Veter Diagn Invest. Official Publication of the American Association of Veterinary Laboratory Diagnosticians, Inc. 2012;24:253-61.

[8] Bolin SR, Grooms DL. Origination and consequences of bovine viral diarrhea virus diversity. Vet Clin North Am Food Anim Pract 2004;20:51-68.

[9] Fulton RW. Impact of species and subgenotypes of bovine viral diarrhea virus on control by vaccination. Anim Health Res Rev 2015;16:40-54.

[10] Hibberd RC, Turkington A, Brownlie J. Fatal bovine viral diarrhoea virus infection of adult cattle. Vet Rec 1993;132:227.

[11] Strong R, La Rocca SA, Paton D, Bensaude E, Sandvik T, Davis L, et al. Viral dose and immunosuppression modulate the progression of acute BVDV-1 infection in calves: evidence of long term persistence after intra-nasal infection. PloS One 2015;10:e0124689.

[12] Liu L, Kampa J, Belak S, Baule C. Virus recovery and full-length sequence analysis of atypical bovine pestivirus Th/04_KhonKaen. Vet Microbiol 2009;138:62-8.

[13] Stahl K, Kampa J, Alenius S, Persson Wadman A, Baule C, Aiumlamai S, et al. Natural infection of cattle with an atypical 'HoBi'-like pestivirus-implications for BVD control and for the safety of biological products. Veter Res 2007:38:517-23.

[14] Meyling A. Detection of BVD virus in viremic cattle by an indirect immunoperoxidase technique. In: McNulty MS JBM, editor. Recent advances in virus diagnosis. Boston, MA: Martinus Nijhoff; 1984. p. 37-46.
15] Larska M, Polak MP, Riitho V, Strong R, Belak S, Alenius S, et al. Kinetics of single and dual infection of calves with an Asian atypical bovine pestivirus and a highly virulent strain of bovine viral diarrhoea virus 1. Comp Immunol, Microbiol Inf Dis 2012;35:381-90.

[16] Coggins L, Gillespie JH, Robson DS, Thompson JD, Phillips WV, Wagner WC, et al. Attenuation of virus diarrhea virus (strain Oregon C24V) for vaccine purposes. Cornell Vet 1961;51:539-45.

[17] Wakeley PR, Turner JL, Ibata G, King DP, Sandvik T, Howard P, et al Characterisation of a type 2 bovine viral diarrhoea virus isolated from cattle in the UK. Vet Microbiol 2004;102:19-24.

[18] Vantsis JT, Barlow RM, Fraser J, Rennie JC, Mould DL. Experiments in Border disease. VIII. Propagation and properties of a cytopathic virus. J Comp Pathol 1976;86:111-20.

[19] Edwards S. The diagnosis of bovine virus diarrhoea-mucosal disease in cattle. Rev Sci Tech 1990;9:115-30.

[20] Ridpath JF, Fulton RW, Kirkland PD, Neill JD. Prevalence and antigenic differences observed between Bovine viral diarrhea virus subgenotypes isolated from cattle in Australia and feedlots in the southwestern United States. J Veter Diagn Invest. Official Publication of the American Association of Veterinary Laboratory Diagnosticians, Inc. 2010;22:184-91.

[21] Riitho V, Walters AA, Somavarapu S, Lamp B, Rumenapf T, Krey T, et al. Design and evaluation of the immunogenicity and efficacy of a biomimetic particulate formulation of viral antigens. Sci Rep 2017;7:13743.

[22] Coad M, Clifford D, Rhodes SG, Hewinson RG, Vordermeier HM, Whelan AO Repeat tuberculin skin testing leads to desensitisation in naturally infected tuberculous cattle which is associated with elevated interleukin-10 and decreased interleukin-1 beta responses. Vet Res 2010;41:14.

[23] Decaro N, Lucente MS, Mari V, Cirone F, Cordioli P, Camero M, et al. Atypical pestivirus and severe respiratory disease in calves, Europe. Emerg Inf Dis 2011;17:1549-52.

[24] Schirrmeier H, Strebelow G, Depner K, Hoffmann B, Beer M. Genetic and antigenic characterization of an atypical pestivirus isolate, a putative member of a novel pestivirus species. J Gen Virol 2004;85:3647-52.

[25] Bauermann FV, Falkenberg SM, Decaro N, Flores EF, Ridpath JF. Experimental infection of calves, sheep, goats and pigs with HoBi-like viruses by direct inoculation or exposure to persistently infected calves. Veter Microbiol 2015;181:289-93.

[26] Bauermann FV, Ridpath JF, Weiblen R, Flores EF. HoBi-like viruses: an emerging group of pestiviruses. J Veter Diagn Invest. Official Publication of the American Association of Veterinary Laboratory Diagnosticians, Inc. 2013;25:6-15

[27] Ridpath JF. Practical significance of heterogeneity among BVDV strains: impact of biotype and genotype on U.S. control programs. Prev. Veter. Med 2005;72:17-30; discussion 215-9.

[28] Kapil S, Walz P, Wilkerson M, Minocha H. In: Immunity and immunosuppression. Bovine viral diarrhea virus. Blackwell Publishing Ltd; 2008. p. 157-70. 